\title{
Notes on Operations Acquisitions Globalized: The Foreign Language Acquisitions Experience in a Research Library
}

\section{By Judit H. Ward}

This paper highlights foreign language titles from the perspective of acquisitions in a large academic research library. Selecting, ordering, cataloging, and providing access to non-English materials reach beyond the boundaries of departments responsible for the individual tasks. Assignments require different levels of language proficiency ranging from bibliographic proficiency to the near-native proficiency of the educated speaker. The highest level of language proficiency is used at the earliest and latest point of technical services (i.e., ordering and cataloging), and the rest requires only bibliographic proficiency or none at all. Because international vendor experiences vary country by country, strong cooperation is critical between the partners in the acquisition process. Vendor-supplied records used for foreign language acquisition purposes seem to have the potential to improve accuracy in bibliographic records.

Foreign language titles have always attracted special attention in North American research libraries because of the uncommon nature of selection and acquisition methods. In an academic and research setting, the dissemination and enhanced access to scholarly information in foreign languages have never been more important than in the current, increasingly globalized information market and in ubiquitous international research cooperation. Changes in research patterns have created a greater diversity of needs and increased demand for foreign titles, and emerging and nontraditional research areas require a broader array of materials.

Although an accurate and up-to-date assessment of North American collections of foreign language materials seems to be difficult to complete at any given time, research has revealed declining foreign language acquisitions in North American academic libraries starting in the 1980s. ${ }^{1}$ The 1985 Research Library Group Study singled out language as the most statistically significant factor in the failure to acquire books. ${ }^{2}$ Other reasons include financial constraints (i.e., the deflation of the dollar against the world currency market and ever rising costs of materials) and political developments that influence collection strategies. ${ }^{3}$ Since the 1990s, foreign language collections in the United States have increasingly been influenced by three main factors: political changes, such as the extraordinary transformations in Eastern Europe, the former Soviet Union, and Latin America; the effect of the global marketplace's growing need for personnel well trained in foreign languages and cultures; and the subsequently expanding boundaries of research, including a remarkable increase in collaboration between researchers in science and technology. ${ }^{4}$

Historically, several projects and programs were established after the foreign materials acquisitions crisis was discovered and documented. Organized and funded by a variety of sources, they aimed at sharing resources and cooperating to facilitate the development of coordinated and distributed collections in research libraries, including special regional and area-based cooperative programs such as the Association of Research Libraries Foreign Acquisitions Project, Seminar for the
Judit H. Ward (judit.ward@rutgers edu) is Director of Information Services, Center of Alcohol Studies, Rutgers, The State University of New Jersey, Piscataway.

Submitted March 26, 2008; returned to author May 3, 2008 with request for revision and resubmission; revised, resubmitted August 25, 2009, and accepted for publication. 
Acquisition of Latin American Library Materials, Latin American Research Resources Project, and the Library of Congress (LC) National Program of Acquisitions and Cataloging. ${ }^{5}$ Many of these initiatives approach cooperative collection development from a fresh, new perspective and with an electronic availability in mind. What all these projects have in common is the need for appropriate human resources in the member libraries, including librarians and paraprofessionals with foreign language skills, as well as a thorough workflow analysis to implement the most effective use of these resources.

\section{Selection and Acquisition: Differences between International and U.S. Imprints}

In addition to the language variations, foreign language acquisitions differ in many ways from domestic acquisitions, which may involve the automated process of the post-selection purchasing of current U.S. imprints with Machine-Readable Cataloging (MARC) records loaded into the integrated library system (ILS). Among the differences are issues with particular languages and language skills of staff, availability and quality of books and bibliographic records, variety in the levels of automation, and diverse library vendor relations. Acquisitions of foreign language titles have always been a problem, regardless of the subject. The complexities of selecting, acquiring, and cataloging foreign language publications can be demonstrated by the developments in the area of Slavic and East European titles, where the methods have not changed significantly from the description published by Burger in $1990 .{ }^{6}$ Selection and acquisition outside of approval plans remained an important way to develop library collections. ${ }^{7}$

In the case of North American publications, much of the selector's work of selecting, rejecting, referring, or saving titles for later selection in large research libraries often happens mostly in the vendor's online database. The database also provides the selector with the history of library activities, prevents duplication, and features direct connection with the module or interface used by the acquisitions staff. Selectors and acquisitions staff may work in the database through the same interface. Once the selector's work has been finished, acquisitions staff members locate and export selected items in the online system and complete orders electronically. As a result, bibliographic records may be loaded overnight into the ILS in an automated process, generating purchase orders title by title. With a less automated method, the acquisition staff member locates the best source to purchase titles and completes the order electronically, which includes exporting or creating records. The possibility of batch processing (batching items to search, order, and load into the ILS significantly reduces turnaround time) allows a smaller margin of errors and excludes accidental duplication. Keeping track of orders, claiming, invoicing, and overriding duplication rules are all provided by the vendor's database. Books, shelfready if requested (i.e., bar-coded, labeled, and tattle-taped), are shipped within a few weeks in sturdy boxes, each containing a packing slip. Most North American vendors work with dependable domestic carriers and usually also use quality shipping and filling material, and books also are often shrink-wrapped individually. The books are received and cataloged by either copy or original catalogers, all trained to perform the given activity in English. Expectations toward international book vendors are defined by this type of acquisition experience.

Similar to the process outlined above, selection of foreign language titles is conducted by librarians, usually subject specialists in a particular field with appropriate language skills. These skills are often gained through studies for another advanced degree or the result of being raised in a bilingual or multilingual environment. Selection methods, however, may largely rely on more conventional sources, such as paper slips, publishers' and book jobbers' print catalogs, prepublication announcements, special offers, or informal channels. Only the major European vendors, such as Aux Amateurs de Livres International and Jean Touzot Librairie Internationale in France, Casalini Libri in Italy, Otto Harrassowitz in Germany, and Puvill Libros in Spain, offer online catalogs with some or all of the options of electronic ordering, invoicing, and providing MARC records. These sites are also available in English, and feature similar functions to major U.S. vendors' password-protected sites and databases, such as order status and statements of account balances. In the case of other languages, the situation is far from ideal from the North American buyer's perspective.

The process of selecting and acquiring titles in foreign languages also may differ significantly by language, country, culture, and vendor. Services are heavily influenced by the vendor's incentive to expand in the North American market and to keep up with the advancements in technical services in the North American libraries. The quality of the books varies significantly from country to country, and print runs are relatively short. ${ }^{8}$ Prices may also fluctuate and may incorporate hefty bank transfer fees for small purchases, which makes planning and budgeting in a library extremely difficult. Shipping might be unpredictable, delayed, or at least nontraceable, and duplicates are costly to return. The quality of packaging may range from the highest quality packing materials to soft boxes of dubious origin with improvised filling material.

Ordering foreign language titles 
requires advanced or at least intermediate language proficiency. After finding the sources of acquisitions, the staff member has to type or load a bibliographic record into the ILS before attaching a purchase order. Acquisition records range from brief records (i.e., author, title, and minimal publication data) to full catalog records exported from a bibliographic utility. Language proficiency at this point is crucial for the subsequent steps in processing the book in hand. Locating the proper record in a bibliographic utility as well as loading or typing appropriate records into the ILS at the point of acquisition can be a time-consuming process. However, the time invested in the first phase is compensated by the smaller margin of error and instant availability. It also makes receiving and copy cataloging books feasible by a staff member with a lower level of foreign language proficiency in the case of non-Roman alphabets and nearly no foreign language proficiency in the case of Roman scripts. Coordinating and organizing the various steps of selection and acquisition will largely depend on available language skills and subject expertise in a particular library setting.

\section{Cooperation as Solution: Vendor-Supplied Bibliographic Records}

Cooperative efforts have assisted libraries in many areas. Beginning in 1996, OCLC and the Research Libraries Group started to load minimal-level catalog records from several European book vendors into their respective databases. Most of the European vendor records in OCLC's WorldCat are from France, Germany, Italy, and Spain. Since five of the ten largest language groups of the world can be found in Europe and are responsible for 44 percent of the world book production, academic libraries collect heavily from these countries. ${ }^{9}$ The respective booksellers in each country took the initiative and created records for the majority of the titles published in their country or in the language of their country. Two booksellers from Spain contribute records to WorldCat: Puvill Libros and Iberbook. Otto Harrassowitz contributes records for German imprints, Casalini Libri provides records for books from Italy, and Jean Touzot Librairie Internationale and Aux Amateurs de Livres supply records for French imprints. By 2000, vendor records accounted for 16.7 percent of Spanish books, 18 percent of French books, 33.6 percent of German books, and 52.5 percent of Italian books. ${ }^{10}$ The number of libraries enhancing vendor records in WorldCat was found only to be approximately one-third of the number of libraries contributing original records for European language books. For example, 60 percent of records for books in Italian were contributed to WorldCat by a vendor, whereas 30 percent were contributed by the LC and only 10 percent by member libraries. ${ }^{11}$

Authors have raised questions about the value of minimal-level vendor catalog records for European language monographs and their effect on catalog department workflows and national cooperative cataloging efforts. ${ }^{12}$ The main feature that distinguishes the vendor records from other minimal-level bibliographic records is that the author, series, and subject headings on the nonvendor records have a much higher likelihood of matching the authorized form for these headings. In contrast, the headings on vendor records, if they are present at all, tend not to match the authoritative form. ${ }^{13}$

Notable initiatives were launched to mitigate these issues in the past few years. Catalogers at Casalini Libri have recently been trained by an LC representative in the LC's classification and subject heading systems. ${ }^{14}$ OCLC's Enhance program (www.nelinet.net/oclc/cataloging/ enhance.htm) was established to encourage participant libraries to upgrade vendor records contributed in non-English languages. This program is designed to allow skilled catalogers to improve the quality of the OCLC WorldCat database by upgrading WorldCat records, primarily from lessthan-full level to full level. The LC developed the MARC Record Guide for Monograph Aggregator Vendors, which was prepared by the Program for Cooperative Cataloging in 2006. ${ }^{15}$

In addition to the efforts for an international cooperation to routinely provide bibliographic records to WorldCat, international vendorsupplied records serve as an example of sensible outsourcing. An article published in 2007 with an extensive review of recent outsourcing experience-albeit not in an international context-strongly advocates an outsourcing program of this kind. ${ }^{16}$ The thorough evaluation of cataloging and physical processing supplied through the University of Arkansas Libraries' shelf-ready contract with YBP Library Services and PromptCat in 2005-6 points out that the workflow is considerably shortened in most cases, and the shelf-ready procedures are briefer than those used for normal copy cataloging.

One of the most recent initiatives, WorldCat Selection Services, is an example of merging the best of collaboration and outsourcing for the benefit of academic and research libraries. Based on the software called the Integrated Tool for Selection and Ordering developed at Cornell University Library, it features an online tool to streamline and automate selection and acquisition with the ability to handle foreign language titles. This tool integrates records from multiple vendors in a single system without differentiating domestic and foreign orders and records. Batch processing also allows loading records into the ILS early in the technical services process. ${ }^{17}$ In another collaborative initiative, the LC, Bibliothèque nationale de France, Deutsche Nationalbibliothek, and OCLC have signed a memorandum of understanding to extend and 
enhance the Virtual International Authority File, a project that virtually combines multiple name authority files into a single name authority service. $^{18}$

Foreign vendor records are valuable from a broader perspective. Not only are they often more accurate than the ad hoc brief record keyed in from an unverified source by a staff member lacking the sufficient language skills, but records in WorldCat often help verify titles and availability. For the acquisitions workflow, minimallevel vendor records can mean greater accuracy and faster turnaround. A significantly lower level of language proficiency is necessary to identify and process these titles. For the local library user, these records provide access to the information at the earliest point of acquisition.

\section{A Case Study: Acquisitions of Foreign Language Titles at Rutgers University Libraries}

The curriculum at Rutgers, the State University of New Jersey, relies heavily on foreign languages both at the undergraduate and graduate levels on all three campuses. In addition to the Master of Arts, Master of Arts in Teaching, and $\mathrm{PhD}$ programs in several languages offered by the respective departments, Rutgers also operates the World Languages Institute, a major outreach graduate-level program for K-12 world language teachers, including the K-12 Chinese Teacher Training Initiative. As a state university, it is also a repository for domestic and foreign government documents.

During the past five years, Rutgers University Libraries (RUL) used various processes. In 2002, the forwardlooking management at the Technical and Automated Services created a new staff position to focus on foreign language acquisitions. This position required language proficiency in at least three European languages, including Russian. The position was filled from 2003 to 2007 by a former linguist and language instructor with advanced-level proficiency in the major European languages, including Russian, and bibliographic proficiency in many more.

Types of acquisition resources vary from language to language and from country to country. The scenario closest to the established RUL practice with current North American imprints is the selection and acquisition of titles in European languages, while RUL had many difficulties with Chinese, Japanese, and Korean (CJK) orders for a long time. The CJK workflow changed several times during these five years, corresponding to the changes in human resources. RUL made significant efforts to blend CJK workflow into the library routine, including selection, ordering, shipping, and tracing books as well as invoice processing. The recently established South Asian Studies Program means new challenges.

Titles in European languages are typically selected by several subject specialists, such as the world history librarian, music librarian, women's studies librarian, and art and humanities librarians, then ordered by the same acquisitions staff member. German, French, and Italian titles have proven to be the easiest to select and order because of the online systems of the respective vendors. These vendors closely follow the trends of the North American market, are present at major North American conferences, and tend to meet the general expectations in the United States in terms of electronic notification, duplication, online catalogs, and ordering, as well as invoicing, claiming, shipping, returns, and rush orders.

\section{Spanish and Portuguese Language Acquisitions}

A large number of the foreign language titles at RUL are monographs in Spanish and Portuguese because of the needs of one of the largest language departments at the university. The Department of Spanish and Portuguese offers both undergraduate and graduate courses in Hispanic literature, culture, and civilization as well as courses in Spanish linguistics, literary theory, Luso-Brazilian literature and culture, translation, interpretation, and teaching and research methodology. The Spanish-English bilingual selector had an incredibly complex and demanding job to meet user needs and ordering deadlines imposed by the fiscal year. Titles were selected from the broadest sources ranging from formal to informal, such as booksellers' print and online notifications, vendors' websites, titles or booklists sent to her by antiquarians or friends, prepublication notes and special offers, and personal e-mail messages and communications, to mention a few. Consequently, the acquisitions department's task was relatively easy for the majority of the titles in terms of verifying the availability with vendors or conducting a preorder search in the local catalog. Unconventional selection methods, however, tested the resourcefulness of the acquisition staff occasionally.

The first serious challenge in foreign language acquisitions is to locate quality bibliographic records to load into the ILS. Using OCLC Connexion (previously OCLC CatME) software does not always bring the desired results. Because many Latin American and European titles might be reprints with or without the same ISBN, individual title search has proven successful in most cases. A title-by-title search instead of a batch ISBN search significantly slowed down the workflow. These records, many of them provided by vendors, could be uploaded to the ILS as a batch simultaneously with the English titles.

The next challenge for the ordering staff member is to find the recently loaded record in the local system so the purchase order could be attached to it (i.e., one order to one title as stipulated by the strict auditing rules 
as well as for convenient follow-up with receiving or claiming). Again, the failure with the simple ISBN searches in the local system does not mean the title is not there. In RUL's experience, typing in a foreign language inadvertently slows down the process and also increases the potential for typos. The lack of the appropriate language proficiency seems to have coincided with a larger number of errors when creating brief records for these titles instead of working with minimal-level vendor records, no matter how embryonic they are.

\section{Ordering in European Languages: French, Italian, and German}

Acquisition of other titles in foreign languages with Roman scripts effortlessly followed the same workflow after the pilot of about three thousand orders of Spanish and Portuguese language titles in 2003. Because of budget allocations, monographs written in lessertaught languages such as Romanian, Hungarian, and Polish were ordered only occasionally and required individual attention. However, frequently requested French, German, and Italian titles were easy to order after the selection, which mostly happened from vendor-supplied data, either print or electronic. French titles at RUL are selected by an art librarian (including topics in French literature, culture, and language) and by the women's studies or music librarian for their respective topics from slips by Aux Amateurs de Livres and Jean Touzot Librairie Internationale. Both vendors provide records to OCLC, the major source of records exports for RUL. The French selector also started to use electronic slips recently, and services that these two vendors offer online are yet to be explored.

Italian titles are selected by another art librarian and purchased from Casalini Libri, the vendor for titles published in Italy or written in Italian. Casalini features integrated library services with its monograph and serial databases (i libri and le riviste) to support selection and acquisitions. The database offers a search platform with advanced search options, such as bibliographic fields, subject fields by call number types, and others complemented by further optional fields such as interdisciplinary topics, level of audience, book types, and special formats. It is also possible to narrow the search to titles recently profiled by Casalini Libri or by price as well as titles on order. Selectors can set up their own profiles, while acquisitions staff have access to orderrelated information and can order online. Similar to major North American vendors, Casalini offers options to create a selection list and save titles including local order data, receive title information and selection lists automatically via e-mail, download bibliographical records in MARC format, and place orders online. Casalini recently expanded the coverage to supply publications in other European languages. Casalini Libri is an excellent example of a vendor that offers a large variety of services and allows libraries to take advantage of them to the degree that best suits their needs. The RUL acquisitions staff ordered from paper notification slips and used the site only occasionally to verify availability and monitor order status. These slips contain the necessary bibliographic information; therefore ordering does not require more than a bibliographic proficiency of Italian. The same level is sufficient for receiving and copy cataloging. After the new foreign language acquisition workflow was implemented for Italian titles, Rutgers had no problems or returns.

German titles are selected by the world history librarian, who has advanced-level language proficiency. The vendor, Otto Harrassowitz, has an online database called OttoEditions, which has been widely used for both selection and acquisition. Technically, online ordering is available for the titles selected by the librarian. However, for workflow and human resources reasons, it is easier for RUL to use this service for rush orders only and to place regular orders according to the workflow streamlined with Spanish and Portuguese titles. Nevertheless, high efficiency applies to the acquisition of German titles selected on the vendor's site or from paper slips and ordered both electronically and in traditional ways. Harrassowitz targets the academic and research library community by specializing in the distribution of scholarly books, periodicals, e-resources, and music scores. This vendor consolidates all German and some European bookselling in periodical subscriptions, databases, standing orders, and music scores. OttoSerials, Harrassowitz's online management system for periodicals and standing orders, simplifies the handling of print and electronic subscriptions for libraries. They can also provide MARC records, while (as a new service to facilitate pre-order searching) any OttoEditions account can be set up to automatically search the library's online catalog from OttoEditions with certain fields appearing as links in the OttoEditions bibliographic display. Clicking on any of these links initiates a search in the library's online catalog, allowing one to determine quickly if a library already holds that item.

\section{Acquisitions in Non-Roman Scripts}

With four members proficient in Russian in RUL Technical and Automated Services, the limited number of Cyrillic orders has never been a problem. Neither have titles in Hebrew or Arabic, with a native speaker at the cataloging department. However, a part-time East Asian librarian and the foreign language acquisitions staff member were experimenting with an array of workflows for CJK orders during a four-year period. Typically, the librarian (a native speaker of Chinese) completed selection and communicated with the vendors located either in the United States or overseas, while the acquisitions staff member placed a 
single batch order attached to a shadow record (i.e., a bibliographic record invisible to patrons). This record contained codes that only these two people were able to decipher at any given time. Another part-time staff member at the cataloging department, a Korean native with strong reading skills in Chinese and Japanese, helped the acquisitions staff with receiving. Because of the nature of CJK resources, books ordered at the same time were rarely shipped in one package, and it was very difficult to keep track of orders. When the order request was filled in several shipments, invoicing became confusing even with excellent records upon receipt. Toward the end of the fiscal year, encumbered funds and orders to be rolled over to the next fiscal year became confusing. Keeping track of a batch of fifty titles or more delivered in four or more shipments over a period of a few weeks was nearly impossible, and RUL completely overlooked the problems that drop shipments (i.e., when orders were completed by an overseas publisher upon the initiation of the vendor) were bound to cause. Even with several adjustments to address the lack of the necessary language skills at the point of acquisition, the workflow looked like a total disaster from the budgeting and invoicing points of view despite the collaboration and RUL's best intentions. The light at the end of the tunnel showed only when a fulltime CJK cataloger librarian was hired with the language skills required for this complex task.

\section{Lessons Learned}

RUL's experience of ordering a large number of foreign language titles from a variety of resources in a period of nearly five years may be helpful for technical services departments of other libraries that need to explore foreign acquisitions workflows. With diminishing resources available, academic libraries face the challenges of designing more effective workflows and may want to experiment with stronger collaboration of librarians, technical services staff, and vendors.

\section{The Effect of Foreign Acquisitions on Workflow and Staffing}

In comparison to English language acquisitions, the workflow to acquire foreign language titles is typically characterized by less automation, longer processing times, and more frequent human intervention. Benchmarks in technical services, usually based on English language titles, do not work with foreign language titles. Problem orders with U.S. imprints, including rush orders, out-of-print books, and special and rare formats and titles frequently have separate workflows in the acquisitions department's daily practice. Additionally, foreign orders may have distinct workflows for each language, depending on language expertise, selection resources, and the availability of titles and bibliographic records. Occasionally special foreign orders, such as audiovisual materials or computer files, may mean an even greater challenge with their compatibility issues.

The acquisitions department at RUL did not have to deal with extreme difficulties of staff turnover during these years. Cross-training allowed scheduling staff members according to demands. There were noticeable differences in the workload in the different phases of the fiscal year, including peak times of ordering followed by an overwhelming number of shipments to be processed. Making the best use of staff time entailed assigning the appropriate level and number of staff to handle the particular demands at any given time. One example is opening boxes shipped to the library, an assignment requiring minimal foreign language skills. In practice, the books are placed on book carts in the same order as they are listed on the packing list. If done correctly, usually by parttime student employees with some foreign language skills, staff members who usually work with English books can match books in hand to the packing slips and can quickly receive and copy catalog titles in any foreign language with Roman scripts.

Clear expectations at each step of the foreign language acquisitions process as well as extensive documentation understandable at each level seem to have helped eliminate anxiety and frustration, an argument often mentioned by staff members in the context of foreign language acquisitions. Creating a departmentwide skills inventory and documenting work processes allowed a more sensible reorganization of the workflow. Simple but creative solutions, such as a problem shelf established at a central location in the technical services building, proved to be helpful. Once staff members realized that they were not supposed to go beyond their responsibilities or abandon their routines for foreign titles, they did not become disappointed so easily. Neither did they distract their coworker in charge of troubleshooting by interrupting them with every single book - they simply placed problematic items on the shelf for later review. The shelf also made it evident and observable that other staff members had problems, too. Troubleshooting was completed by the same staff member who made the first step in the workflow, which allowed a deeper insight into the nature of the issues. In the long run, this practice yielded a thorough analysis of errors and resulted in the elimination of many of them. Handling problems in batches revealed patterns and intrinsically suggested solutions. Assigning one person to solve problems also avoided the duplication of efforts in this area. The optimal workflow, streamlined with Spanish and Portuguese titles, allowed the foreign language acquisitions staff member to dedicate her time entirely to language-specific tasks because the number of errors and returns dramatically dropped to less than 3 percent. 
Vendor-supplied bibliographic records (available in the Research Libraries Group's union catalog at that time and from OCLC later) have played a role in reviewing and reorganizing the workflow, making Spanish and Portuguese language acquisitions more efficient.

RUL's case shows that with proactive management, consolidating foreign language acquisition workflows and integrating the process into the daily routine in a large academic library setting is feasible. The redesign of the foreign language workflow was endorsed by the head and assistant head of the acquisitions department, and the supportive environment provided by Technical and Automated Services also greatly facilitated changes by encouraging resource sharing, cooperation, and training. Another factor worth mentioning was revamping intra- and interdepartmental communication, such as consolidating staff notes and records about problems or regularly inviting acquisitions staff members to cataloging group meetings. Identifying the components of the foreign language acquisition process from a larger perspective worked for the Technical and Automated Services staff members and encouraged them to find their role in accomplishing departmental goals. Foreign titles have proven to build bridges in many ways within and outside the library.

\section{Language Proficiency Needed}

As far as particular foreign language skills are concerned, the acquisitions department at RUL had the most difficulty with CJK orders. The issues originated when selection, acquisition, and end-processing happened at the East Asian Library, where the librarian and the staff had the proper language expertise to select and process the books in the library, but separately from the Technical and Automated Services workflow. However, invoicing and claiming-and especially following up with problems - caused a significant challenge for the acquisitions department and the budget office. After the retirement of the East Asian librarian, several attempts were made to overcome residual issues and integrate CJK ordering workflow into the general acquisition process in light of the successful workflow with other languages. Without the necessary language skills at the acquisitions department, CJK orders became nightmarish. Japanese titles were the only exceptions; these were selected by a librarian with the appropriate language skills and e-mailed to both the vendor and the acquisitions staff, the latter placing a batch order. The success was the result of the excellent communication and strong relationship with a reputable vendor. A willingness to comply with the expectations at North American libraries is remarkable in the case of all international vendors. Without the high level of vendor cooperation, completing many of these orders would have been nearly impossible.

The CJK acquisitions experience highlights the necessity of bibliographic proficiency of staff members at the acquisitions department. Russian and other Cyrillic scripts presented no problems because more than one librarian and more than one acquisition and cataloging staff member had these language skills ranging from basic reading skills, such as the ability to verify whether the book in hand matches the book ordered, to more advanced, such as strong reading skills or near-native proficiency. Regarding the level of language skills required at an acquisitions department, basic language proficiency seems to be working, provided the selector and the cataloger at previous and subsequent points in the process are able to provide support. Although linguistic abilities are frequently listed within cultural competencies, library schools do not have any language requirement nor do they offer special training for language-related librarian positions or copious language-related tasks in a library in public and technical services.

The Spanish and Portuguese acquisition workflow at RUL can serve as a prime example that, at the initial point of acquisition, the language proficiency of the staff member who locates records and orders the titles is crucial for the subsequent steps. Loading or keying in a proper record is vital to avoid duplication and costly returns, and also enables staff members with no or limited Spanish language proficiency to match the book in hand to the order and invoice upon receipt, which results in a significant increase of efficiency and a dramatic decrease in time spent troubleshooting. In comparison with the workflow of current U.S. imprints, verifying the availability of proper records and identifying them are the responsibilities of an experienced staff member, whereas the fairly routine part of receiving is done by employees in clerical positions. The term proper record may not be satisfactory for cataloging purposes and is used here to denote a variety of bibliographic records with several access points for the user while complying with the minimum requirements of an acquisition record.

\section{Collaboration: Selectors, Acquisitions Staff, and Vendors}

The acquisition of foreign language titles has been considered a central issue at RUL. Identifying barriers and bottlenecks in the workflow of foreign language acquisitions from a broader perspective allowed RUL to learn and encouraged RUL to experiment with various methods of collaboration using a variety of communication strategies. In the case of many languages, the collaboration among all participants (i.e., vendor, acquisitions staff, cataloging staff, and selector) resulted in significantly faster turnaround times and fewer mistakes. Vendor performance 
was assessed and continuously monitored, which led to the creation of an in-house vendor database based on the selection sources. Improved communication also ensured that all participants of the acquisitions process were on the same page concerning the level of automation in a particular language. As pointed out before, the workflow of ordering Russian language titles was seamless for two main reasons: sufficient language proficiency at all points and the excellent collaboration between the vendors and the library, including the selector, acquisitions and cataloging staff, and the library budget office.

Whenever available, vendorsupplied records were used for foreign language titles acquisitions to verify data and availability. In most cases, the advantages of having an easily available and mostly correct record balanced the inaccuracies of the record. The acquisitions record served as a source to keep track of orders both by the acquisitions staff and the selectors, as a starting point for catalogers to upgrade the record, and as an access point for reference librarians and library users while the title was still at an early phase in Technical and Automated Services. Few libraries can afford to hire librarians and staff members with extensive skills in every language at all service points and in all departments, but collaboration is available for all.

\section{The Foreign Language Acquisitions Experience}

In comparison to North American acquisitions, the foreign language vendor experience is not better or worse, just different. Favorable experiences far outweigh the complications. Troubleshooting is considered one of the main criteria to evaluate vendor services, and most foreign language vendors match to or even surpass domestic vendors in this area. Not only do they respond to problems in a timely manner with creative solutions, but they also demonstrate a noticeably high level of responsibility and accountability. Another important criterion of evaluation, handling order requests out of the ordinary workflow, can also be pointed out as one of the strongest points of many foreign language vendors. Foreign language acquisitions are full of difficulties. The challenge is to be resourceful and always ready to overcome linguistic, cultural, physical, and communication barriers.

\section{References}

1. "Acquisition and Distribution of Foreign Language and Area Studies Materials," Journal of Library Administration 29, no. 3/4 (2000): 51-75; "Research Libraries in a Global Context: An Exploratory Paper," Journal of Library Administration 29, no. 3 (2000): 77-91.

2. "Research Libraries in a Global Context," 83.

3. "Acquisition and Distribution of Foreign Language and Areas Studies Materials."

4. "Research Libraries in a Global Context."

5. Patricia Brennan and Jutta ReedScott, Cooperative Strategies in Foreign Acquisitions, SPEC Flyer no. 195 (Washington, D.C.: Association of Research Libraries, Office of Management Services, 1993).

6. Robert Burger, "Slavic Technical Services," Technical Services Today and Tomorrow, ed. Michael Gorman (Littleton, Colo.: Libraries Unlimited, 1990): 130-41.

7. Keren Dali and Juris Dilevko, "Beyond Approval Plans: Methods of Selection and Acquisition of Books in Slavic and East European Languages in North American Libraries," Library Collections, Acquisitions, \& Technical Services 29, no. 3 (Sept. 2005): 23869.

8. Sue Henczel, "Selecting and Acquiring Library Materials in Languages Other than English: Establishing NonEnglish Collections for Public, School and Academic Libraries," Collection Building 22, no. 3 (2003): 141-45.
9. Knut Dorn, "Champagne Taste and a Beer Budget: The Problem of Increasing Scholarly Publishing in Europe and Decreasing Academic Library Budgets in North America" (paper presented at the annual meeting of the Center for Research Libraries, Chicago, Apr. 24, 1992).

10. Charlene Kellsey, "Cooperative Cataloging, Vendor Records, and European Language Monographs," Library Resources \& Technical Services 46, no. 3 (July 2002): 10510.

11. Charlene Kellsey, "Trends in Source of Catalog Records for European Monographs 1996-2000," Library Resources \& Technical Services 45, no. 3 (July 2001): 123-26.

12. Laura D. Shedenhelm and Bartley A. Burk, "Book Vendor Records in the OCLC Database: Boon or Bane?" Library Resources \& Technical Services 45, no. 1 (Jan. 2001): 10-19.

13. Jeffrey Beall, "The Impact of Vendor Records on Cataloging and Access in Academic Libraries," Library Collections, Acquisitions, \& Technical Services 24, no. 2 (Summer 2000): 229-37.

14. Kellsey, "Trends in Source of Catalog Records."

15. Library of Congress, Program for Cooperative Cataloging, MARC Record Guide for Monograph Aggregator Vendors, www.loc.gov/ catdir/pcc/sca/FinalVendorGuide.pdf (accessed Mar. 19, 2008).

16. Mary Walker and Deb Kulczak, "Shelf-Ready Books using PromptCat and YBP: Issues to Consider," Library Collections, Acquisitions, \& Technical Services 31, no. 2 (June 2007): 61-84.

17. OCLC, "WorldCat Selection Service," webinar presented Feb. 2007, www5 .oclc.org/downloads/wcsp/default.htm (accessed Mar. 19, 2008).

18. Library of Congress, "News from the Library of Congress: Library of Congress, Bibliothèque nationale de France, Deutsche Nationalbibliothek and OCLC Enhance VIAF Project" press release, Nov. 16, 2007, www .loc.gov/today/pr/2007/07-236.html (accessed Mar. 19, 2008). 